\title{
Trends in Endovascular Reperfusion Therapy for Acute Stroke after Introduction of Mechanical Thrombectomy Devices: Japanese Registry of NeuroEndovascular Therapy (JR-NET)3
}

\author{
Mikito HaYaKawa, ${ }^{1}$ Yuji Matsumaru, ${ }^{1,2}$ Hiroshi Yamagami, ${ }^{3}$ Tetsu Satow, ${ }^{4}$ \\ Koji IIHARA, ${ }^{5}$ Nobuyuki SAKAI, ${ }^{6}$ and JR-NET investigators \\ ${ }^{1}$ Division of Stroke Prevention and Treatment, Faculty of Medicine, University of Tsukuba, \\ Tsukuba, Ibaraki, Japan; \\ ${ }^{2}$ Department of Neurosurgery, Faculty of Medicine, University of Tsukuba, Tsukuba, \\ Ibaraki, Japan; \\ ${ }^{3}$ Department of Stroke Neurology, National Hospital Organization Osaka National Hospital, \\ Osaka, Osaka, Japan; \\ ${ }^{4}$ Department of Neurosurgery, National Cerebral and Cardiovascular Center, Suita, \\ Osaka, Japan; \\ ${ }^{5}$ Department of Neurosurgery, Graduate School of Medical Sciences Kyusyu University, \\ Fukuoka, Fukuoka, Japan; \\ ${ }^{6}$ Department of Neurosurgery, Kobe City Medical Center General Hospital, Kobe, \\ Hyogo, Japan
}

\begin{abstract}
In Japan, the era of thrombectomy for acute stroke started when the Merci retriever was approved in 2010 and the Penumbra system in 2011. New-generation Penumbra catheters (MAX series) and stent retrievers were introduced in 2013 and 2014, respectively. The aim of this study was to clarify the general status and trends in endovascular reperfusion therapy for stroke in Japan from 2010 to 2014 . We extracted a total of 3705 patients $(72.1 \pm 12.2$ years, 1482 women) from a nationwide study of patients receiving any neuroendovascular treatment, the Japanese Registry of NeuroEndovascular Therapy 3. Reperfusion therapy resulted in a successful reperfusion rate of $59 \%$, and $31 \%$ of patients achieved a favorable outcome at 30 days of onset. The frequency of Merci-including procedures increased to $52 \%$ in the first half of 2011, but conversely decreased to $1 \%$ in the second half of 2014 . The frequency of Penumbra-including procedures increased from $18 \%$ in the second half of 2011 to $67 \%$ in the first half of 2014, decreasing to $38 \%$ in the second half of 2014. The reperfusion rate and 30-day clinical outcomes remained almost unchanged until June 2013, and started to improve thereafter, coincident with the introduction and spread of newer-generation devices. The rate of successful reperfusion reached $71-76 \%$, and $36-38 \%$ of patients achieved a favorable outcome in 2014. After multivariate adjustment for preprocedure variables, the calendar period from 2013 to 2014 was significantly associated with improvement in reperfusion rates and clinical outcomes.
\end{abstract}

Key words: acute ischemic stroke, large vessel occlusion, endovascular therapy, nationwide survey, trend

\section{Introduction}

Since 2015, mechanical thrombectomy using stent retrievers and/or large-bore aspiration catheters for

Received November 27, 2019; Accepted December 20, 2019

Copyright $\odot 2020$ by The Japan Neurosurgical Society This work is licensed under a Creative Commons AttributionNonCommercial-NoDerivatives International License. acute stroke patients with large vessel occlusion has been proven to improve patients' clinical outcomes, ${ }^{1-111}$ and has currently become the "standard of care". ${ }^{12)}$

In Japan, the era of thrombectomy for acute stroke started when the Merci retriever (Concentric Medical, Mountain View, CA, USA) was approved in April 2010 (launched in October 2010). ${ }^{13)}$ Subsequently, the Penumbra system (Penumbra Inc., Alameda, CA, USA) was approved in June 2011 (launched in October 
2011). In addition, the therapeutic window from symptom onset for intravenous thrombolysis using recombinant tissue-plasminogen activator (IV rt-PA) was extended to $4.5 \mathrm{~h}$ in August 2012. ${ }^{14)}$ In June 2013, new-generation Penumbra aspiration catheters, the MAX series, was introduced in routine clinical practice. The Solitaire FR (Covidien, Mansfield, MA, USA) and Trevo XP ProVue (Stryker, Kalamazoo, MI, USA) stent retrievers were approved in December 2013 and March 2014, respectively, and both were reimbursable by Japanese health insurance systems since July 2014. The Penumbra 5MAX ACE was subsequently introduced in October 2014.

The Japanese Registry of NeuroEndovascular Therapy (JR-NET) study group conducted retrospective studies in three consecutive phases, to clarify the general status of neuroendovascular therapy performed by specialists certified by the Japanese Society for NeuroEndovascular Therapy (JSNET): JR-NET, was performed from January 2005 to December 2006, JR-NET2 from January 2007 to December 2009, and JR-NET3 from January 2010 to December 2014. ${ }^{15,16)}$ We previously reported on the trends of endovascular therapy (EVT) for acute stroke due to large vessel occlusion between 2005 and 2009, equivalent to the "pre-Merci" era in Japan, using JR-NET/JR-NET2 datasets, and showed that EVT at that time mainly consisted of intra-arterial thrombolysis (IAT) and percutaneous transluminal balloon angioplasty (PTA)/balloon clot disruption (BCD) (stand-alone IAT, 37.4\%; IAT + PTA/BCD, $27.4 \%$; and stand-alone PTA/BCD, $16.0 \%) .{ }^{17}$

The study period of the JR-NET3 encompassed the era from introduction of first-generation thrombectomy devices (Merci retriever and the initial devices of the Penumbra system) to the early spread of stent retrievers, but before establishment of the efficacy of EVT for acute stroke in improving clinical outcomes. The general status of EVT for acute stroke at that time had not yet been reported comprehensively. The aim of this study was to clarify the trends in baseline characteristics, procedures, reperfusion status and clinical outcomes of acute stroke patients with large vessel occlusion who underwent EVT after introduction of mechanical thrombectomy devices in Japan.

\section{Materials and Methods}

The JR-NET3 was a Japanese nationwide retrospective study and registered a total of 40,177 patients who received any neuroendovascular treatment by JSNET-certified neurointerventionalists at 166 centers from January 2010 to December 2014. ${ }^{16)} \mathrm{We}$ extracted acute stroke patients who underwent EVT from the registry. The Institutional Review Board at each center approved the retrospective use of patient data. Consent to participate in this study was obtained using an opt-out approach.

We summarized the baseline characteristics [age, sex, premorbid modified Rankin Scale (mRS) score, baseline National Institutes of Health Stroke Scale (NIHSS) score, stroke subtypes, culprit occluded arteries, baseline Alberta Stroke Program Early Computed Tomography Score (ASPECTS)/ASPECTS $+\mathrm{W}^{18}$ ], imaging modalities [CT and magnetic resonance imaging (MRI)], onset-to-treatment time (OTT) and revascularization procedures (EVT with or without preceding IV rt-PA, stand-alone IAT, stand-alone PTA/BCD, stand-alone stenting, standalone Merci thrombectomy, stand-alone Penumbra thromboaspiration, stand-alone clot retrieval using retrievers other than the Merci retriever, other single procedures, and procedural combinations) performed in these patients. Similarly, we evaluated the rates of successful reperfusion, defined as a Thrombolysis in Cerebral Infarction (TICI) grade of $2 \mathrm{~b}-3$, and complete reperfusion, defined as a TICI grade of 3 . We also evaluated procedure-related intracranial hemorrhagic complications with any clinical deterioration within $24 \mathrm{~h}$ of EVT and clinical outcomes at 30 days of stroke onset. A favorable outcome was defined as a mRS score of $0-2$. Finally, we analyzed the semiannual trends in baseline characteristics, use of IV rt-PA, EVT procedures, and the treatment results.

The culprit occluded arteries were differentiated into the internal carotid artery [ICA, including simultaneous middle cerebral artery (MCA) and/ or other vessel occlusion], the MCA-M1 (including simultaneous occlusion of its branches), MCA-M2 (including simultaneous occlusion of its branches), basilar artery (BA, including simultaneous vertebral and/or posterior cerebral artery occlusion), and other arteries.

Intravenous thrombolysis using recombinant tissue-plasminogen activator was performed using $0.6 \mathrm{mg} / \mathrm{kg}$ of alteplase within a 3-h window (up to August 2012) or a 4.5-h window (from September 2012) from symptom onset, according to the standard protocol approved in Japan, ${ }^{14)}$ and EVT procedures were performed according to the protocol at each center by a JSNET-certified specialist physician or operator.

Reperfusion status was angiographically evaluated by the physician at each center at the end of EVT. The mRS score at hospital discharge was used as a substitute for outcome when 30-day outcome data was unavailable.

Data were expressed as a proportion, mean value \pm standard deviation (SD) or median value [interquartile range $(\mathrm{IQR})]$. For the semi-annual transition 
analyses, we used the Cochrane-Armitage trend test or Jonckheere-Terpstra trend test, as appropriate. Furthermore, we used Fisher's exact test or Kruskal-Wallis test with Bonferroni correction for multiple comparisons to investigate differences between two consecutive half-years.

In addition, we assessed the associations between calendar time and study endpoints (successful/ complete reperfusion and 30-day clinical outcome) using multiple logistic regression analysis adjusted for preprocedural variables, including age, sex, premorbid mRS score, large-artery atherosclerosis, occlusion of ICA or BA, baseline NIHSS score, OTT, and IV rt-PA use (Model 1), for preprocedural variables and successful reperfusion (Model 2), and for preprocedural variables, successful reperfusion and intracranial hemorrhagic complications (Model 3) by the forced-entry method. Models 2 and 3 were applied only to 30-day clinical outcomes. A $P$-value $<0.05$ was considered statistically significant.

Data were statistically analyzed using IBM SPSS Statistics 24 software (IBM SPSS, Chicago, IL, USA) and EZR (Saitama Medical Center, Jichi Medical University, Saitama, Japan), ${ }^{19)}$ a graphical user interface for R, version 3.5.2 (R Foundation for Statistical Computing, Vienna, Austria).

\section{Results}

\section{Study population and patient characteristics}

Among 3897 acute stroke patients who underwent EVT in the JR-NET3 dataset, a total of 3705 patients $(72.1 \pm 12.2$ years, 1482 women $)$ were analyzed in this study after exclusion of 171 cases with duplicated data and 21 cases with incomplete data on age, sex and/or treatment information. Table 1 shows the patients' baseline characteristics and diagnostic imaging modalities. The proportion of patients who were premorbidly independent was $89.3 \%$. The median baseline NIHSS score was 18 (IQR, 12-23), and the leading stroke subtype was cardioembolic stroke in $70.2 \%$. The culprit occluded arteries were the ICA in $31.2 \%$, MCA-M1 in $40.0 \%$ and BA in $13.1 \%$. MRI, including MR angiography was performed before the procedure in $91.9 \%$ of patients. The OTT was $\leq 3 \mathrm{~h}$ in $37.8 \%, 3-4.5 \mathrm{~h}$ in $27.3 \%$, and $4.5-6 \mathrm{~h}$ in $17.3 \%$ of the patients. In patients with an anterior-circulation large-vessel occlusion, the median baseline ASPECTS and ASPECTS + W were 9 (IQR, 7-10) and 8 (IQR, 6-9), respectively.

\section{Revascularization procedures}

Details of the revascularization procedures performed are noted in Table 2. IV rt-PA was administered in $37.7 \%$ of patients prior to EVT. The single-most frequent EVT procedure was Penumbra thromboaspiration in $21.3 \%$, and second most frequent procedure was Merci thrombectomy in $11.4 \%$. Regarding procedure combinations, Penumbra-including procedure combinations were most frequently used in $39.5 \%$, as with single procedures, while the second most frequent procedure combinations included PTA/ BCD in $26.3 \%$.

\section{Treatment results}

Table 2 shows the results of treatment in the study subjects. Successful reperfusion was achieved in $59.2 \%$ and complete reperfusion in $27.4 \%$ of the patients. The rate of intracranial hemorrhagic complications was $9.6 \%$. Clinical outcomes were favorable in $30.5 \%$, with fatalities in $11.1 \%$ at 30 days of stroke onset.

\section{Semi-annual trends in patient characteristics}

Table 1 also shows the semi-annual transitions of patient baseline characteristics and imaging modalities. In the trend of the number of cases, it increased steadily from 172 in the first half (H1) of 2010 to 560 in the second half (H2) of 2014. While patient age gradually increased throughout the study period $(70.4 \pm 12.2$ years in H1 2010 to $73.4 \pm 11.9$ years in H2 2014, $P$ for trend $<0.001$ ), no significant trends were observed in baseline NIHSS score ( $P$ for trend $=0.319$ ), baseline ASPECTS $(P$ for trend $=0.154)$ or baseline ASPECTS $+\mathrm{W}(P$ for trend $=0.746)$. The proportion of cardioembolic strokes increased from $63.4 \%$ to $72.7 \%$ ( $P$ for trend $=0.045$ ), whereas the proportion of large-artery atherosclerosis decreased from $24.4 \%$ to $16.1 \%$ ( $P$ for trend $<0.001$ ) over the 5-year study period. Although there was no significant trend of the proportion of ICA occlusion ( $P$ for trend $=0.789$ ) or MCA-M1 occlusion ( $P$ for trend $=0.350$ ), the proportion of BA occlusion decreased from $25.7 \%$ in $\mathrm{H} 12010$ to $13.6 \%$ in H2 2010, remaining almost flat thereafter, although the incidence of MCA-M2 occlusion increased from $9.8 \%$ in $\mathrm{H} 22013$ to $15.5 \%$ in H2 2014. These trends were statistically significant throughout the 5 years (BA, $P$ for trend $=0.001$; MCA-M2, $P$ for trend $=0.009$ ). The frequency of preprocedural MRI evaluations increased to the $94 \%$ range in $\mathrm{H} 2$ 2012, remaining at the same level into H1 2014 ( $P$ for trend $=0.031$ ). It, however, decreased to $90.5 \%$ in H2 2014. The proportion of patients with OTT within $3 \mathrm{~h}$ declined from $36 \%$ in $\mathrm{H} 1$ to $26 \%$ in $\mathrm{H} 2$ 2010 , increasing steadily thereafter throughout the remaining observation period to $42 \%$ ( $P$ for trend $<0.001)$. On the other hand, the proportion of patients with OTT of $4.5-6 \mathrm{~h}$ gradually decreased from $20 \%$ to $14 \%$ over the 5 years ( $P$ for trend $=0.034$ ). 


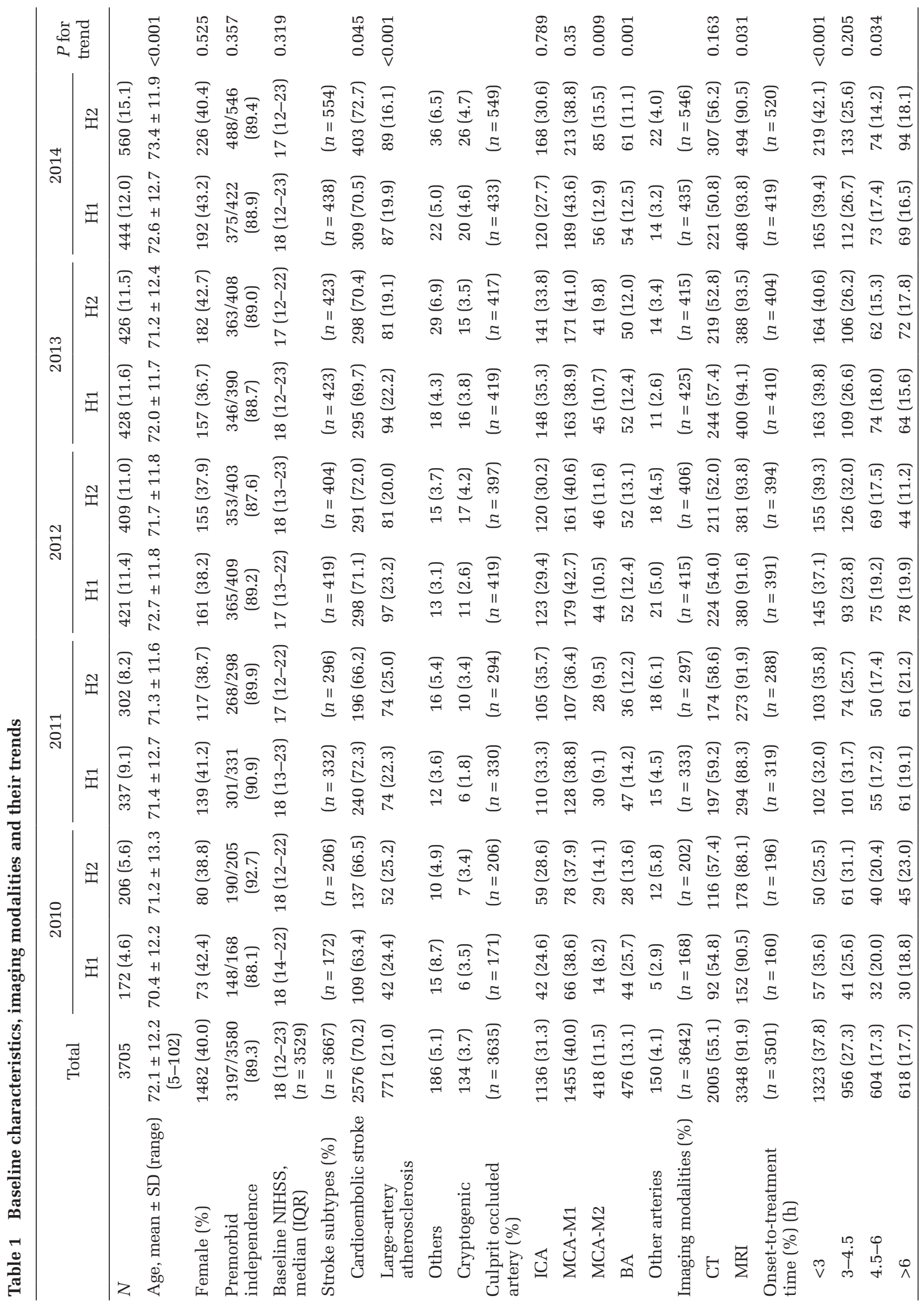




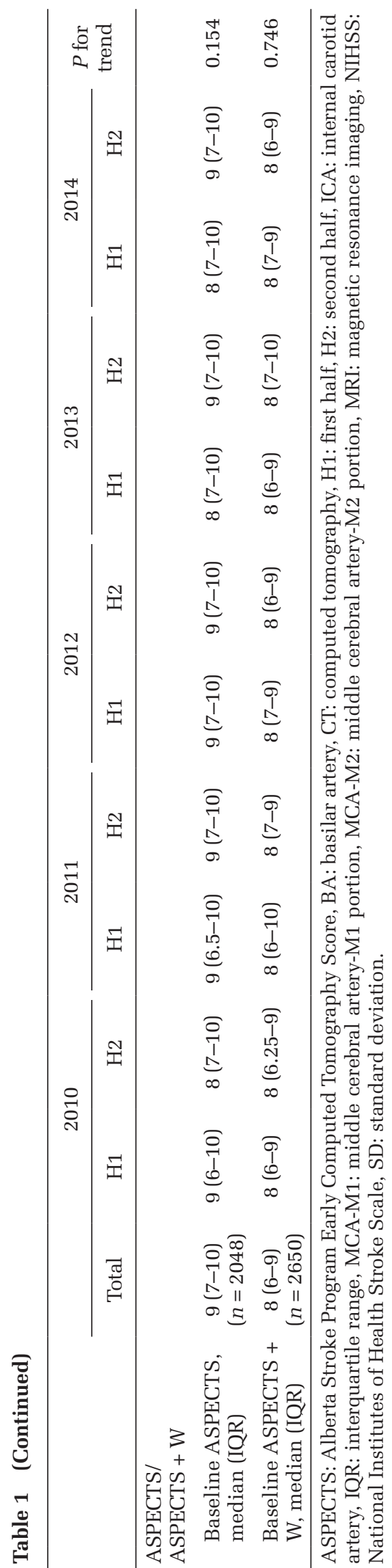

Table 2 Revascularization procedures and treatment results

\begin{tabular}{|c|c|}
\hline & Total \\
\hline$N$ & 3705 \\
\hline IV rt-PA (\%) & $1346 / 3572(37.7)$ \\
\hline EVT procedures (\%) & $(n=3676)$ \\
\hline Stand-alone IAT & $359(9.8)$ \\
\hline Stand-alone PTA/BCD & $332(9.0)$ \\
\hline $\begin{array}{l}\text { Stand-alone thrombectomy using } \\
\text { the Merci retriever }\end{array}$ & $420(11.4)$ \\
\hline $\begin{array}{l}\text { Stand-alone thromboaspiration } \\
\text { using the Penumbra catheter }\end{array}$ & $783(21.3)$ \\
\hline $\begin{array}{l}\text { Stand-alone thrombectomy using } \\
\text { other than the Merci retriever }\end{array}$ & $236(6.4)$ \\
\hline Stand-alone stenting & $141(3.8)$ \\
\hline Other single procedures & $102(2.8)$ \\
\hline Any procedural combinations & $1303(35.4)$ \\
\hline Any combination including IAT & $884(24.0)$ \\
\hline Any combination including PTA/BCD & $966(26.3)$ \\
\hline $\begin{array}{l}\text { Any combination including the } \\
\text { Merci retriever }\end{array}$ & $888(24.2)$ \\
\hline $\begin{array}{l}\text { Any combination including the } \\
\text { Penumbra catheter }\end{array}$ & $1452(39.5)$ \\
\hline $\begin{array}{l}\text { Any combination including } \\
\text { thrombectomy devices other than } \\
\text { the Merci retriever }\end{array}$ & $377(10.3)$ \\
\hline Any combination including stenting & $426(11.6)$ \\
\hline Successful reperfusion (\%) & $2166 / 3657(59.2)$ \\
\hline TICI 3 & $1001(27.4)$ \\
\hline $\begin{array}{l}\text { Procedure-related intracranial } \\
\text { hemorrhagic complications }\end{array}$ & $353 / 3692(9.6)$ \\
\hline Clinical outcome at 30 days (\%) & $(n=3608)$ \\
\hline Favorable & $1100(30.5)$ \\
\hline Death & $402(11.1)$ \\
\hline
\end{tabular}

EVT: endovascular therapy, IAT: intra-arterial thrombolysis, IV rt-PA: intravenous recombinant tissue-plasminogen activator, PTA/BCD: percutaneous transluminal angioplasty/ balloon clot disruption, TICI: Thrombolysis in Cerebral Infarction.

\section{Semi-annual trends in revascularization procedures and treatment results}

Figure 1 shows the trends of revascularization procedures and treatment results. The proportion of patients receiving IV rt-PA showed a straightforward increase from $19.6 \%$ in $\mathrm{H} 12010$ to $45.3 \%$ in $\mathrm{H} 2$ 2013, just after the approval of the wider therapeutic time window of $4.5 \mathrm{~h}$ ( $P$ for trend $<0.001$, Fig. $1 \mathrm{~A})$. Simultaneously, the frequencies of EVT procedures, including IAT and PTA/BCD, decreased abruptly from $50.3 \%$ and $64.9 \%$ in $\mathrm{H} 12010$ to $6.9 \%$ and 

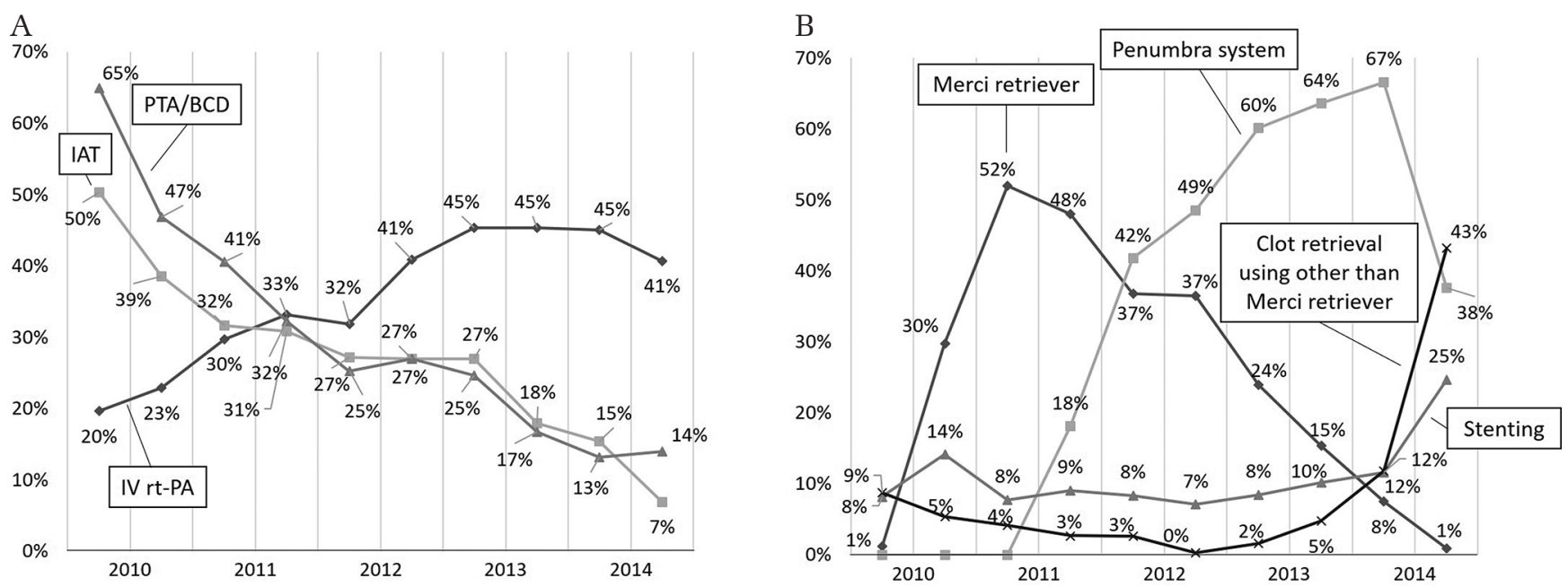

C

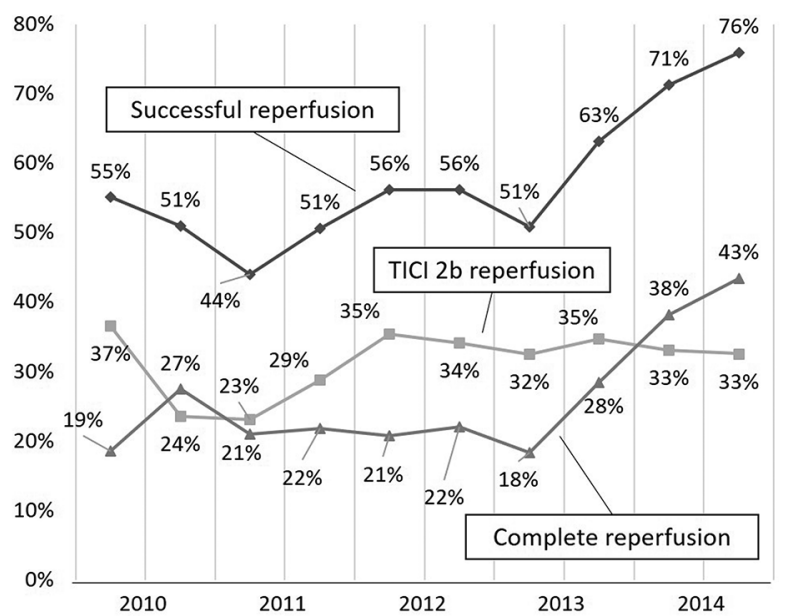

$\mathrm{D}$

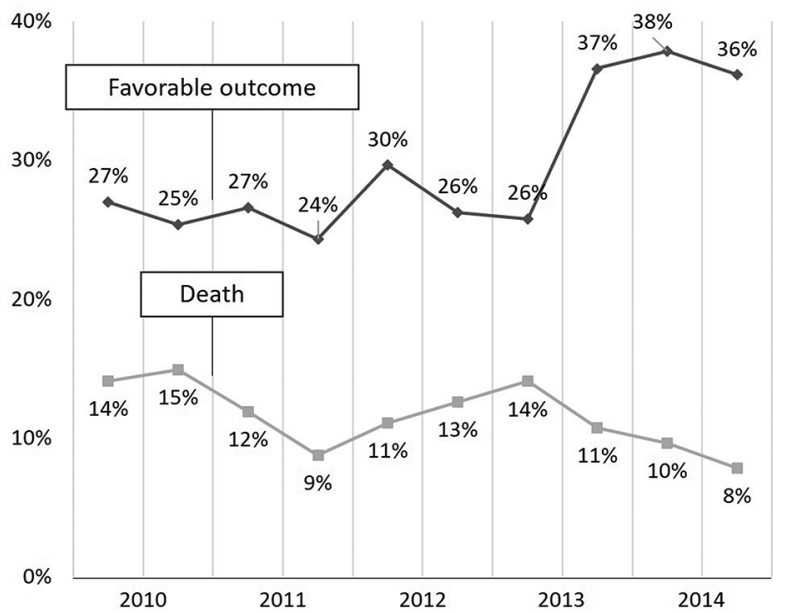

Fig. 1 Trends in revascularization procedures and treatment results. (A) Intravenous thrombolysis using recombinant tissue-plasminogen activator, intra-arterial thrombolysis and percutaneous transluminal angioplasty/ balloon clot disruption. (B) Mechanical procedures other than percutaneous transluminal angioplasty/balloon clot disruption. (C) Reperfusion status. (D) Clinical outcomes. IAT: intra-arterial thrombolysis, IV rt-PA: intravenous recombinant tissue-plasminogen activator, PTA/BCD: percutaneous transluminal angioplasty/balloon clot disruption, TICI: Thrombolysis in Cerebral Infarction.

$14.0 \%$ in H2 2014, respectively ( $P$ for trend of both $<0.001$, Fig. 1A).

Figure 1B shows the trends of mechanical procedures other than PTA/BCD. The frequency of Merci-including procedures abruptly increased from $1.2 \%$ in $\mathrm{H} 12010$ to $51.9 \%$ in $\mathrm{H} 22011$, decreasing thereafter to $0.9 \%$ in $\mathrm{H} 22014$. The Penumbra device was first used in $\mathrm{H} 1$ 2011, and the frequency of Penumbra-including procedures increased to $66.6 \%$ in $\mathrm{H} 12$ 2014. However, it decreased to $37.6 \%$ in $\mathrm{H} 2$ 2014. The frequency of any procedure combination including thrombectomy devices other than the Merci retriever began to increase in H2 2013, reaching $43.2 \%$ in $\mathrm{H} 22014$ ( $P$ for trend $<0.001$, $P$ between H2 2013 and H1 $2014=0.008, P$ between the halves of $2014<0.001)$. Similarly, the frequency of all procedure combinations that included stenting acutely doubled from $11.6 \%$ in $\mathrm{H} 1$ to $24.7 \%$ in $\mathrm{H} 2$ 2014 ( $P$ for trend $<0.001, P$ between the halves of $2014<0.001)$.

The rate of successful reperfusion decreased from $55.1 \%$ in $\mathrm{H} 12010$ to $44.0 \%$ in $\mathrm{H} 12011$, increasing again to $56.2 \%$ in 2012. In H1 2013, it modestly decreased to $50.8 \%$, followed by a steady increase until the end of the study period ( $P$ for trend $<0.001$, Fig. 1C). There was, in particular, a significant increase in successful reperfusion rates between the two halves of $2013(P=0.017)$. The rate of TICI $2 \mathrm{~b}$ reperfusion, however, increased from $23.1 \%$ in H1 2011 to $35.4 \%$ in H1 2012 and remained 
flat thereafter ( $P$ for trend $=0.018$ ). The rate of complete reperfusion remained at or slightly above 20\% from H1 2011 to H1 2013, and then, rapidly increased to $43.4 \%$ in $\mathrm{H} 22014$, in line with the rate of successful reperfusion $(P$ for trend $<0.001$, $P$ between the halves of $2013=0.027)$.

The trend of patients with a fatal outcome throughout the study period decreased from $14.1 \%$ to $7.8 \%$, with modest fluctuations ( $P$ for trend $=0.01$, Fig. 1D). The proportion of patients with a favorable outcome remained almost unchanged at a little under $30 \%$ through the start of the study period up to H1 2013, but increased up to 36.1-37.8\% in H2 2013 and 2014 ( $P$ for trend $<0.001, P$ between the halves of $2013=0.043)$. The rate of procedure-related intracranial hemorrhagic complications showed no interval change throughout the study period ( $P$ for trend $=0.627$ ).

\section{Relationships between calendar time and study endpoints}

Table 3 shows the relationships between calendar time and each study endpoint. Multiple logistic regression analyses revealed that calendar time was significantly associated with each study endpoint after adjustment for preprocedural variables (Model 1): successful reperfusion [odds ratio (OR), 1.159; 95\% confidence interval (CI), 1.125-1.193], complete reperfusion (OR, 1.164; 95\% CI, 1.126-1.202), favorable outcome (OR, $1.077 ; 95 \%$ CI, 1.042-1.140) and death (OR, 0.954; 95\% CI, 0.911-0.999). When dividing the study period into two phases, 2010-2012 as the first-generation-device period and 2013-2014 as the newer-generation-device (the Penumbra MAX series and stent retrievers) period, the degree of chronological improvement in the rate of successful reperfusions was greater in the later phase (OR, 1.578; 95\% CI, 1.426-1.746) than in the earlier phase (OR, 1.109; 95\% CI, 1.037-1.185). Furthermore, chronological improvements in the rate of complete reperfusion or 30-day clinical outcomes were statistically significant in the later phase but not in the earlier phase.

When adding successful reperfusion (Model 2) or successful reperfusion and intracranial hemorrhagic complications (Model 3) to the variables in Model 1, the relationships between calendar time and clinical outcomes became insignificant, except for mortality in the later phase.

Table 3 Adjusted Odds ratio with 95\% confidence interval per half year for each study endpoint

\begin{tabular}{|c|c|c|c|c|c|c|}
\hline & \multicolumn{2}{|l|}{ Model $1^{*}$} & \multicolumn{2}{|l|}{ Model $2^{+}$} & \multicolumn{2}{|l|}{ Model $3^{\ddagger}$} \\
\hline & Odds ratio (95\% CI) & $P$-value & Odds ratio (95\% CI) & $P$-value & Odds ratio (95\% CI) & $P$-value \\
\hline \multicolumn{7}{|l|}{ Successful reperfusion } \\
\hline 2010-2014 (per half year) & $1.159(1.125-1.193)$ & $<0.001$ & - & - & - & - \\
\hline 2010-2012 (per half year) & 1.109 (1.037-1.185) & 0.002 & - & - & - & - \\
\hline 2013-2014 (per half year) & $1.578(1.426-1.746)$ & $<0.001$ & - & - & - & - \\
\hline \multicolumn{7}{|l|}{ Complete reperfusion } \\
\hline 2010-2014 (per half year) & $1.164(1.126-1.202)$ & $<0.001$ & - & - & - & - \\
\hline 2010-2012 (per half year) & $1.012(0.934-1.097)$ & 0.767 & - & - & - & - \\
\hline 2013-2014 (per half year) & $1.606(1.446-1.783)$ & $<0.001$ & - & - & - & - \\
\hline \multicolumn{7}{|l|}{ Favorable outcome } \\
\hline 2010-2014 (per half year) & $1.077(1.042-1.114)$ & $<0.001$ & $1.026(0.991-1.063)$ & 0.151 & $1.029(0.993-1.066)$ & 0.118 \\
\hline 2010-2012 (per half year) & $1.09(0.932-1.093)$ & 0.824 & $0.967(0.888-1.053)$ & 0.444 & $0.982(0.900-1.070)$ & 0.672 \\
\hline 2013-2014 (per half year) & $1.227(1.103-1.366)$ & $<0.001$ & $1.075(0.958-1.206)$ & 0.217 & $1.078(0.960-1.212)$ & 0.204 \\
\hline \multicolumn{7}{|c|}{ Death } \\
\hline 2010-2014 (per half year) & $0.954(0.911-0.999)$ & 0.044 & $0.981(0.935-1.028)$ & 0.418 & $0.978(0.932-1.027)$ & 0.378 \\
\hline 2010-2012 (per half year) & $0.946(0.852-1.051)$ & 0.303 & $0.950(0.852-1.060)$ & 0.359 & $0.920(0.823-1.028)$ & 0.142 \\
\hline 2013-2014 (per half year) & $0.767(0.656-0.896)$ & 0.001 & $0.826(0.703-0.971)$ & 0.02 & $0.814(0.692-0.957)$ & 0.013 \\
\hline
\end{tabular}

${ }^{*}$ Adjusted for preprocedural variables including age, sex, premorbid modified Rankin Scale score, large-artery atherosclerosis, occlusion of internal carotid artery or basilar artery, baseline National Institutes of Health Stroke Scale score, onset-to-treatment time, and intravenous recombinant tissue-plasminogen activator. ${ }^{\dagger}$ Adjusted for preprocedural variables and successful reperfusion. ${ }^{\ddagger}$ Adjusted for preprocedural variables, successful reperfusion and intracranial hemorrhagic complications. CI: confidence interval. 


\section{Discussion}

This study is the largest Japanese nationwide survey of EVT for acute stroke enrolling approximately 3700 patients that clearly showed the trends in revascularization procedures and treatment results after introduction of mechanical thrombectomy devices. The major findings of this study were as follows: the number of patients with advanced age, treated within $3 \mathrm{~h}$ of onset, with cardioembolic stroke, and with MCA-M2 occlusion had been increasing along with an increase in the number of patients registered, while stroke severity remained high, as indicated by median baseline NIHSS scores of 17 or 18 , and infarcted area remained limited, as shown by median ASPECTS or ASPECTS + W of 8 or 9, during the study period; the main thrombectomy devices used changed chronologically with their introduction; the rates of successful/complete reperfusion and the proportion of patients with a favorable/fatal outcome did not improve until June 2013 despite introduction of the Merci retriever in 2010, the Penumbra system in 2011 and extension of the time window of IV rt-PA up to $4.5 \mathrm{~h}$ in 2012, although these outcomes clearly started to improve from June 2013.

\section{Trends of patient characteristics}

In this study, the proportion of patients with OTT $<3 \mathrm{~h}$ chronologically increased up to $42.1 \%$ despite almost all patients being selected based on MRI, which is generally recognized as a timeconsuming selection method compared with CT. ${ }^{20,21)}$ Sakai et al. ${ }^{13)}$ reported time metrics of the initial Japanese experience with the Merci retriever, including 119 patients from 15 centers from 2010 to 2011, showing that the median onset-to-door time was $95 \mathrm{~min}$, median door-to-puncture time was $105 \mathrm{~min}$, and median onset-to-puncture time was $235 \mathrm{~min}$. In the "stent retriever" era, Ota et al. ${ }^{22)}$ showed that the median onset-to-door time was $111 \mathrm{~min}$, median door-to-puncture time was $80 \mathrm{~min}$, and median onset-to-puncture time was $201 \mathrm{~min}$ in the Tama-Registry of Acute Endovascular Thrombectomy (TREAT), a Japanese regionwide survey regarding EVT for acute stroke conducted from 2015 to 2017. Similarly, Yoshimura et al. ${ }^{23)}$ showed that the median onset-to-door time was $120 \mathrm{~min}$ and median onset-to-puncture time was $200 \mathrm{~min}$ in a large prospective cohort study of acute stroke patients with large vessel occlusion, the Recovery by Endovascular Salvage for Cerebral Ultra-Acute Embolism (RESCUE) Japan Registry 2, conducted from 2014 to 2017. According to these studies, which indicated no apparent shortening of the onset-to-door time between the "Merci" and the "stent-retriever" eras, shortening of the door-topuncture time could contribute to faster initiation of EVT. The trend of OTT in the present study might, therefore, reflect sophistication of initial hospital management (workflow) rather than advancements in the patient delivery system. Improvement of in-hospital workflow might allow more patients with cardioembolic stroke, the subtype that tends to progress faster into large/profound cerebral ischemia, to be included in the EVT-eligible population (e.g. severe neurological status but limited infarct area). Furthermore, physicians' experiences with EVT might widen its indications to more elderly patients and more peripheral lesions (e.g. MCA-M2 occlusion).

\section{Trends of revascularization procedures and treatment results}

The frequencies of EVT procedures, including PTA/BCD or IAT, which were mainstream in the "pre-Merci" era, ${ }^{17)}$ abruptly decreased after introduction of the Merci retriever in 2010, with the Merci retriever also being subsequently replaced by the Penumbra system from 2011. There was, however, no obvious improvement in the actual rate of successful reperfusion between 2010 and H1 2013. When adjusted for preprocedural variables, a significant semi-annual improvement in the rate of successful reperfusions (adjusted OR, 1.109; 95\% CI, 1.037-1.185) was revealed. These results might reflect the effects of the learning curve after introduction of first-generation devices. On the other hand, clinical outcomes did not improve over that time, as shown by the unadjusted and multivariate analyses shown in Fig. 1D and Table 3. Hassan et al. ${ }^{24)}$ compared clinical outcomes between the "Merci" era (2004-2007) and "Penumbra" era (2008-2009) in the United States, using the Nationwide Inpatients Sample data files provided by the Agency for Healthcare Research and Quality. Although in-hospital mortality rate tended to decrease in the "Penumbra" era compared with the "Merci" era, the rate of non-mild disability at discharge did not improve. The authors presumed the cause of the unchanged rate of non-mild disability at discharge to be due to chronological increment of futile recanalizations in line with an increase of elderly patients. Futile recanalization similarly might play a role in unchanged clinical outcomes in the present study. An increase in elderly patients, however, seemed unlikely to be related to futile recanalization in our study because multivariate analysis in Model 1 was adjusted for variables including age. A relatively long procedure time using first-generation devices (1.6-2.1 h reported in previous studies $)^{25-27)}$ could be the cause of futile recanalization. 
Unadjusted analysis showed an increase in the proportion of patients with favorable outcomes and a decrease in mortality rate since 2013, in line with an increase in the rate of successful or complete reperfusions. Especially, the treatment results in 2014 compared favorably with the TREAT ${ }^{22}$ (rate of successful reperfusion, $78.8 \%$, and rate of 3 -month independence, $39.6 \%$ ) and RESCUE Japan Registry 2 results $^{23)}$ (rate of 3-month independence, 41.7\%).

Chronological increase in the achievement rate of a favorable outcome was clearly shown by multivariate analysis adjusted for preprocedural variables (Model 1), but the significance was diminished when adjusted for successful reperfusion (Models 2 and 3). Successful reperfusion, therefore, was presumed to strongly affect achievement of a favorable outcome. In Japan, physicians probably started to perform "a direct aspiration first pass technique" (ADAPT, the first paper regarding which was published online in April 2013 ${ }^{28}$ ) at the same time as introduction of the Penumbra Max series in June 2013. Subsequently, stent retrievers (Solitaire and Trevo) and the Penumbra 5MAX ACE became available in 2014. These newergeneration devices/techniques (with a learning curve) could be strongly associated with improvements in clinical outcomes due to the higher and faster reperfusion ability with lower complication risk as compared with first-generation devices. ${ }^{29-31)}$ In fact, the rate of complete reperfusion, which was reported to correlate with a larger outcome-improvement effect than TICI $2 \mathrm{~b}$ reperfusion, ${ }^{32-34)}$ abruptly increased from $18 \%$ in $\mathrm{H} 12013$ to $43 \%$ in $\mathrm{H} 2$ 2014, and the rate of achieving a favorable outcome seemed to increase as a function of an increase in the rate of complete reperfusion. Menon et al. ${ }^{35}$ analyzed data from the Get with the Guideline-Stroke program and showed a significant chronological improvement in clinical outcomes in patients who underwent EVT since 2011, probably due to introduction of newergeneration devices, as in the present study.

Interestingly, the relationship between calendar time from 2013 to 2014 and mortality was significant even in Models 2 and 3. Improvement of in-hospital workflow, patient selection and periprocedural patient management might have been strongly associated with the decrease in mortality at that time, in addition to successful and faster reperfusion.

\section{Limitations}

This study has several limitations. First, this study was retrospective, and some data were missing in enrolled patients. Second, no data of time intervals of in-hospital workflow, such as imaging, treatment initiation, and achieving reperfusion was collected.
Third, it was unclear whether "conventional aspiration with separator" or "ADAPT" was performed for patients undergoing EVT using the Penumbra system. Fourth, the frequency of stent retriever usage could not be determined because these cases were counted as "clot retrieval using other than the Merci retriever" or "stenting" in this study. We, therefore, could not reveal the actual effects of newer-generation devices/techniques on successful reperfusion and clinical outcomes. Lastly, the capture rate of patients was presumed to be approximately $40 \%$ of all procedures performed in Japan. ${ }^{16)}$ We are planning the next JR-NET4 study, which will collect data regarding newer-generation device/technique usage, in-hospital workflow, and other information. This planned study will give accurate information on the effects of newer-generation devices/techniques and improvement in workflow on clinical outcomes in patients with acute large vessel occlusion who underwent EVT in a real clinical setting in Japan.

\section{Conclusion}

In the period of the JR-NET3 study of patients with acute stroke who underwent endovascular reperfusion therapy from 2010 (the time of introduction of mechanical thrombectomy) to 2014, EVT for acute stroke resulted in a rate of successful reperfusion of $59.2 \%$, and $30.5 \%$ of patients achieved a favorable outcome at 30 days of onset. The rate of reperfusion and 30-day clinical outcomes remained almost unchanged until June 2013, subsequently starting to improve from July 2013, coincident with the introduction and spread of newer-generation devices/techniques.

\section{Acknowledgments}

The JR-NET3 Study Group: Co-Principle investigators: Nobuyuki Sakai, Kobe City Medical Center General Hospital, Kobe, Japan; Koji Iihara, Kyushu University, Fukuoka, Japan; Tetsu Satow, National Cerebral and Cardiovascular Center, Suita, Japan; Investigators: Masayuki Ezura, Sendai Medical Center, Sendai, Japan; Akio Hyodo, Dokkyo Medical University Saitama Medical Center, Koshigaya, Japan; Shigeru Miyachi, Aichi Medical University, Aichi, Japan; Susumu Miyamoto, Kyoto University, Kyoto, Japan; Yoji Nagai, Kobe University, Kobe, Japan; Kunihiro Nishimura, National Cerebral and Cardiovascular Center, Suita, Japan; Kazunori Toyoda, National Cerebral and Cardiovascular Center, Suita, Japan; Co-investigators: Toshiyuki Fujinaka, Osaka National Hospital, Osaka, Japan; Toshio Higashi, Fukuoka University, Fukuoka, Japan; 
Masaru Hirohata, Kurume University, Kurume, Japan; Akira Ishii, Kyoto University, Kyoto, Japan; Hirotoshi Imamura, Kobe City Medical Center General Hospital, Kobe, Japan; Yasushi Ito, Shinrakuen Hospital, Niigata, Japan; Naoya Kuwayama, Toyama University, Toyama, Japan; Hidenori Oishi, Juntendo University, Tokyo, Japan; Yuji Matsumaru, Tsukuba University, Tsukuba, Japan; Yasushi Matsumoto, Kohnan Hospital, Sendai, Japan; Ichiro Nakahara, Fujita Medical University, Aichi, Japan; Chiaki Sakai, Hyogo College of Medicine, Nishinomiya, Japan; Kenji Sugiu, Okayama University, Okayama, Japan; Tomoaki Terada, Showa University Fujigaoka Hospital, Kanagawa, Japan; Shinichi Yoshimura, Hyogo College of Medicine, Nishinomiya, Japan; and Certified Specialist of Japanese Society of Neuroendovascular Therapy.

\section{Funding}

This study was supported in part by a Grant-inAid (Junkanki-Kaihatsu H24-4-3) from the National Cerebral and Cardiovascular Center, Japan and by Hatazaki Foundation, Kobe, Japan.

\section{Conflicts of Interest Disclosure}

The authors declare that there are no conflicts of interest.

All authors who are members and non-members of the Japan Neurosurgical Society (JNS) have registered self-reported COI disclosure statements through the JNS website.

\section{References}

1) Berkhemer OA, Fransen PS, Beumer D, et al.: A randomized trial of intraarterial treatment for acute ischemic stroke. N Engl J Med 372: 11-20, 2015

2) Goyal M, Demchuk AM, Menon BK, et al.: Randomized assessment of rapid endovascular treatment of ischemic stroke. N Engl J Med 372: 1019-1030, 2015

3) Campbell BC, Mitchell PJ, Kleinig TJ, et al.: Endovascular therapy for ischemic stroke with perfusionimaging selection. N Engl J Med 372: 1009-1018, 2015

4) Saver JL, Goyal M, Bonafe A, et al.: Stent-retriever thrombectomy after intravenous t-PA vs. t-PA alone in stroke. $N$ Engl J Med 372: 2285-2295, 2015

5) Jovin TG, Chamorro A, Cobo E, et al.: Thrombectomy within 8 hours after symptom onset in ischemic stroke. N Engl J Med 372: 2296-2306, 2015

6) Bracard S, Ducrocq X, Mas JL, et al.: Mechanical thrombectomy after intravenous alteplase versus alteplase alone after stroke (THRACE): a randomised controlled trial. Lancet Neurol 15: 1138-1147, 2016
7) Muir KW, Ford GA, Messow CM, et al.: Endovascular therapy for acute ischaemic stroke: the Pragmatic Ischaemic Stroke Thrombectomy Evaluation (PISTE) randomised, controlled trial. J Neurol Neurosurg Psychiatry 88: 38-44, 2017

8) Nogueira RG, Jadhav AP, Haussen DC, et al.: Thrombectomy 6 to 24 hours after stroke with a mismatch between deficit and infarct. $N$ Engl J Med 378: 11-21, 2018

9) Albers GW, Marks MP, Kemp S, et al.: Thrombectomy for stroke at 6 to 16 hours with selection by perfusion imaging. N Engl J Med 378: 708-718, 2018

10) Lapergue B, Blanc R, Gory B, et al.: Effect of endovascular contact aspiration vs stent retriever on revascularization in patients with acute ischemic stroke and large vessel occlusion: the ASTER randomized clinical trial. JAMA 318: 443-452, 2017

11) Turk AS, Siddiqui A, Fifi JT, et al.: Aspiration thrombectomy versus stent retriever thrombectomy as first-line approach for large vessel occlusion (COMPASS): a multicentre, randomised, open label, blinded outcome, non-inferiority trial. Lancet 393: 998-1008, 2019

12) Powers WJ, Rabinstein AA, Ackerson T, et al.: 2018 Guidelines for the early management of patients with acute ischemic stroke: a Guideline for Healthcare Professionals From the American Heart Association/ American Stroke Association. Stroke 49: e46-e110, 2018

13) Sakai N, Ueda T, Hayakawa M, et al.: Periprocedural results of mechanical thrombectomy using Merci Retriever: Initial experience at Japanese top 15 centers. J Neuroendovasc Ther 5: 23-31, 2011 (Japanese)

14) Minematsu K, Toyoda K, Hirano T, et al.: Guidelines for the intravenous application of recombinant tissue-type plasminogen activator (alteplase), the second edition, October 2012: a guideline from the Japan Stroke Society. J Stroke Cerebrovasc Dis 22: 571-600, 2013

15) Sakai N, Yoshimura S, Taki W, et al.: Recent trends in neuroendovascular therapy in Japan: analysis of a nationwide survey-Japanese Registry of Neuroendovascular Therapy (JR-NET) 1 and 2. Neurol Med Chir (Tokyo) 54: 1-8, 2014

16) Sakai N, Uchida K, Iihara K, et al.: Japanese Surveillance of Neuroendovascular Therapy in JR-NET - Part II. Japanese Registry of NeuroEndovascular Treatment 3. Main Report. Neurol Med Chir (Tokyo) 59: 106-115, 2019

17) Hayakawa M, Yamagami H, Sakai N, Matsumaru Y, Yoshimura S, Toyoda K: Endovascular treatment of acute stroke with major vessel occlusion before approval of mechanical thrombectomy devices in Japan: Japanese Registry of Neuroendovascular Therapy (JR-NET) and JR-NET 2. Neurol Med Chir (Tokyo) 54: 23-31, 2014

18) Kawano $H$, Hirano $T$, Nakajima $M$, Inatomi $Y$, Yonehara T, Uchino M: Modified ASPECTS for 
DWI including deep white matter lesions predicts subsequent intracranial hemorrhage. J Neurol 259: 2045-2052, 2012

19) Kanda Y: Investigation of the freely available easyto-use software 'EZR' for medical statistics. Bone Marrow Transplant 48: 452-458, 2013

20) Menon BK, Almekhlafi MA, Pereira VM, et al.: Optimal workflow and process-based performance measures for endovascular therapy in acute ischemic stroke: analysis of the Solitaire FR thrombectomy for acute revascularization study. Stroke 45: 2024-2029, 2014

21) Goyal M, Jadhav AP, Bonafe A, et al.: Analysis of workflow and time to treatment and the effects on outcome in endovascular treatment of acute ischemic stroke: results from the SWIFT PRIME randomized controlled trial. Radiology 279: 888-897, 2016

22) Ota T, Shigeta K, Amano T, et al.: Regionwide retrospective survey of acute mechanical thrombectomy in Tama, Suburban Tokyo: a preliminary report. J Stroke Cerebrovasc Dis 27: 3350-3355, 2018

23) Yoshimura S, Sakai N, Uchida K, et al.: Endovascular therapy in ischemic stroke with acute largevessel occlusion: recovery by endovascular salvage for cerebral ultra-acute embolism Japan Registry 2. J Am Heart Assoc 7: pii: e008796, 2018

24) Hassan AE, Chaudhry SA, Grigoryan M, Tekle WG, Qureshi AI: National trends in utilization and outcomes of endovascular treatment of acute ischemic stroke patients in the mechanical thrombectomy era. Stroke 43: 3012-3017, 2012

25) Smith WS, Sung G, Starkman S, et al.: Safety and efficacy of mechanical embolectomy in acute ischemic stroke: results of the MERCI trial. Stroke 36: 1432-1438, 2005

26) Smith WS, Sung G, Starkman S, et al.: Mechanical thrombectomy for acute ischemic stroke: final results of the Multi MERCI trial. Stroke 39: 1205-1212, 2008

27) Penumbra Pivotal Stroke Trial Investigators: The penumbra pivotal stroke trial: safety and effectiveness of a new generation of mechanical devices for clot removal in intracranial large vessel occlusive disease. Stroke 40: 2761-2768, 2009
28) Turk AS, Spiotta A, Frei D, et al.: Initial clinical experience with the ADAPT technique: a direct aspiration first pass technique for stroke thrombectomy. J Neurointerv Surg 6: 231-237, 2014

29) Saver JL, Jahan R, Levy EI, et al.: Solitaire flow restoration device versus the Merci Retriever in patients with acute ischaemic stroke (SWIFT): a randomised, parallel-group, non-inferiority trial. Lancet 380: 1241-1249, 2012

30) Nogueira RG, Lutsep HL, Gupta R, et al.: Trevo versus Merci retrievers for thrombectomy revascularisation of large vessel occlusions in acute ischaemic stroke (TREVO 2): a randomised trial. Lancet 380: 1231-1240, 2012

31) Turk AS, Frei D, Fiorella D, et al.: ADAPT FAST study: a direct aspiration first pass technique for acute stroke thrombectomy. J Neurointerv Surg 6: 260-264, 2014

32) Yoo AJ, Simonsen CZ, Prabhakaran S, et al.: Refining angiographic biomarkers of revascularization improving outcome prediction after intra-arterial therapy. Stroke 44: 2509-2512, 2013

33) Kaesmacher J, Dobrocky T, Heldner MR, et al.: Systematic review and meta-analysis on outcome differences among patients with TICI2b versus TICI3 reperfusions: success revisited. J Neurol Neurosurg Psychiatry 89: 910-917, 2018

34) Tung EL, McTaggart RA, Baird GL, et al.: Rethinking thrombolysis in cerebral infarction $2 \mathrm{~b}$ : which thrombolysis in cerebral infarction scales best define near complete recanalization in the modern thrombectomy era? Stroke 48: 2488-2493, 2017

35) Menon BK, Saver JL, Goyal M, et al.: Trends in endovascular therapy and clinical outcomes within the nationwide Get With The Guidelines-Stroke registry. Stroke 46: 989-995, 2015

Address reprint requests to: Mikito Hayakawa, MD, Division of Stroke Prevention and Treatment, Faculty of Medicine, University of Tsukuba, 1-1-1 Tennodai, Tsukuba, Ibaraki 305-8575 Japan. e-mail: mikito-h@jc4.so-net.ne.jp 